\title{
miRNA-877-5p inhibits malignant progression of prostate cancer by directly targeting SSFA2
}

\author{
Wanchun Wang, ${ }^{1}$ Jun Yi, ${ }^{1}$ Degang Dong, ${ }^{2}$ Wenli Mao, ${ }^{1}$ Xuanyu Wang, ${ }^{1}$ Zhangren Yan ${ }^{1}$ \\ ${ }^{1}$ Department of TCM Surgery, Affiliated Hospital of Jiangxi University of Traditional Chinese Medicine, Nanchang; \\ ${ }^{2}$ School of life sciences, Jiangxi University of Traditional Chinese Medicine, Nanchang, China
}

\begin{abstract}
In this study, we aimed to investigate the role of miR-877-5p in the malignant phenotypes of prostate cancer (PCa) cells and its underlying mechanism. RT-qPCR analysis was performed to examine the expression of miR877-5p and sperm-specific antigen 2 (SSFA2) in PCa tissues and cells. Cell counting kit-8 (CCK-8) assay, 5ethynyl-20-deoxyuridine (EdU) assay, flow cytometry, wound-healing assay, and transwell invasion assay were performed to determine the functional roles of miR-877-5p in PCa cells. The association of miR-877-5p with SSFA2 was determined by luciferase reporter and RNA pull-down assays. In this study, we found that the expression level of miR-877-5p was decreased in PCa tissues and cells. Functionally, overexpression of miR877-5p exerted tumor suppressor properties in PCa cells. Mechanistically, SSFA2 was identified as a target gene of miR-877-5p, while overexpression of SSFA2 could abrogate the anti-tumor effects of miR-877-5p in PCa cells. These findings demonstrated that miR-877-5p/SSFA2 axis functioned as a potential target for PCa treatment.
\end{abstract}

Key words: prostate cancer; miR-877-5p; proliferation; migration; invasion.

Correspondence: Zhangren Yan, Department of TCM Surgery, Affiliated Hospital of Jiangxi University of Traditional Chinese Medicine, Nanchang, 330000, China. Tel.+86.13970045572. E-mail: 57671388@qq.com

Contribution: WW, JY, contribute equally to this study. ZY, conceived the study and designed the experiments; WW, JY, completed the experiment, analyzed the data and wrote the manuscript; DD, WM, XW, discussed the results and revised the manuscript.

Funding: This work was supported by National Natural Science Foundation of China (No. 81460730, 81960873).

Conflict of interest: The authors declare that they have no competing interests.

Availability of data and materials: The datasets used and/or analyzed during the current study are available from the corresponding author on reasonable request.

Ethical Approval: Ethical approval was obtained for all experimental procedures by the Ethical Committee of the Affiliated Hospital of Jiangxi University of Traditional Chinese Medicine.

Patient consent for publication: Verbal informed consent was obtained from the patients for their anonymized information to be published in this article. 


\section{Introduction}

Prostate cancer $(\mathrm{PCa})$ is one of the most common malignancies in the urinary system, since its high morbidity and mortality. ${ }^{1}$ Although advances have been achieved in the surgery and chemotherapy, as well as the adjuvant therapy, ${ }^{2-4}$ the prognosis of PCa patients still remains unsatisfactory. ${ }^{5}$ Hence, it is of great urgency to illustrate the specific molecular mechanisms underlying the incidence and development of PCa.

MicroRNAs (miRNAs) are non-coding RNAs containing 18 22 nucleotides, which regulate expressions of target genes by specific binding to the 3'-untranslated region (3' UTR) of the target messenger RNA (mRNA). ${ }^{6}$ miRNAs have been reported to be involved in various cellular processes, including cell proliferation, apoptosis, differentiation and invasion. ${ }^{7-9}$ It has been reported that various miRNAs were aberrantly expressed in different types of cancers, including PCa. ${ }^{6,10,11}$ For instance, miR-34a suppressed the PCa stem cells and metastasis by repressing CD44. ${ }^{12}$ In addition,
miR-3648 facilitates the proliferation via inhibiting adenomatous polyposis coli 2 in PCa cells. ${ }^{13}$ miR-515-5p was demonstrated to serve as a tumor suppressor by targeting TRIP13 in PCa. ${ }^{14}$

A growing body of evidence suggests that miR-877-5p plays essential roles in the tumorigenesis of cancers, including ovarian cancer, ${ }^{15}$ colorectal cancer,${ }^{16}$ hepatocellular carcinoma (HCC), ${ }^{17-19}$ glioblastoma. ${ }^{20}$ and so on. For example, lncRNA DSCAM-AS1 promotes the cervical cancer by targeting miR-877-5p/ATXN7L3 axis. ${ }^{21}$ Moreover, $\operatorname{lncRNA}$ TRG-AS1 has been reported to facilitate glioblastoma cell proliferation by miR-877-5p/SUZ12 axis. ${ }^{20}$ In addition, miR-877-5p suppresses cell growth, migration and invasion by targeting cyclin dependent kinase 14 and predicts prognosis in hepatocellular carcinoma. ${ }^{19}$

Nevertheless, the potential role of miR-877-5p in the progression of $\mathrm{PCa}$ is still unclear. Therefore, we designed the current study to investigate the functions of miR-877-5p in the regulation of PCa cell growth and metastasis, and to propose a novel miRNAmRNA interaction network in PCa progression.
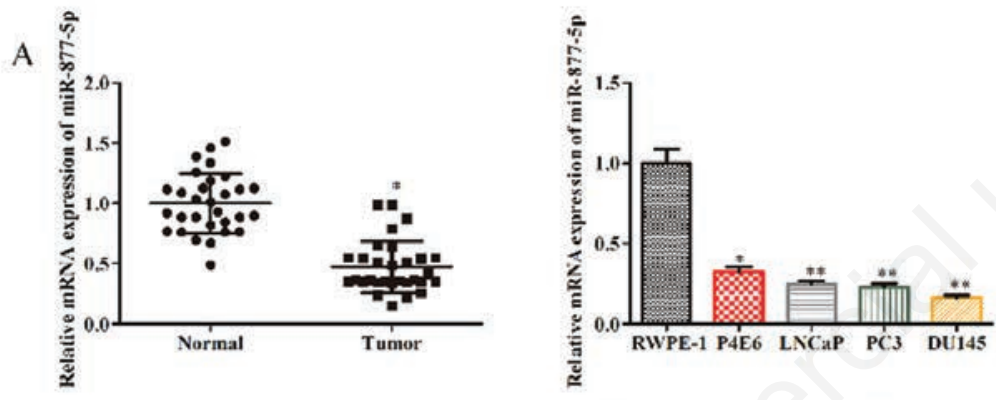

C
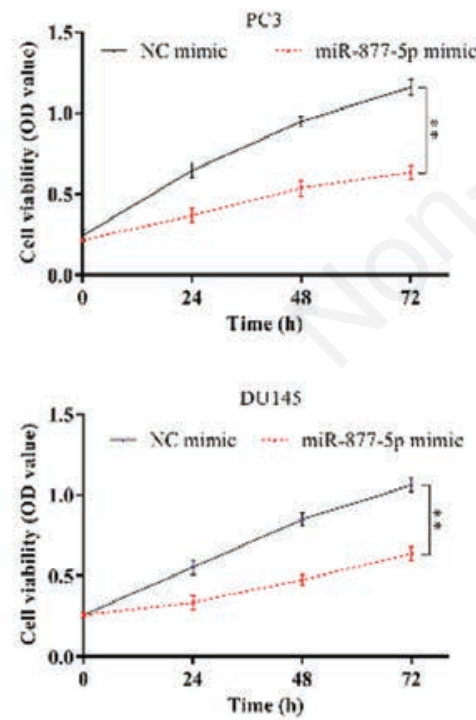

B
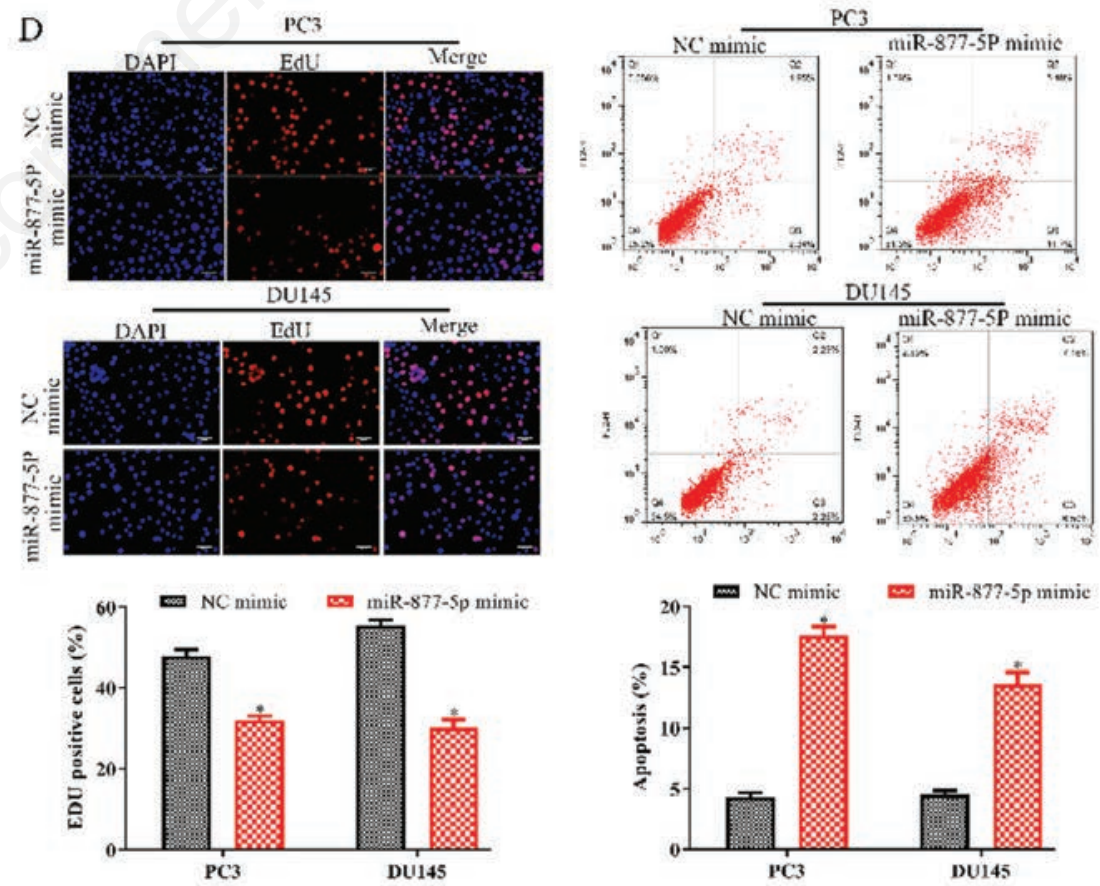

Figure 1. MiR-877-5p suppresses PCa cell proliferation and induces apoptosis. A,B) MiR-877-5p expression at mRNA level in PCa tissues and cell lines were detected using RT-qPCR; ${ }^{*} \mathrm{p}<0.05,{ }^{*} \mathrm{p}<0.01 v$ sWPE-1 cells. B) The transfected efficient was verified using RT-qPCR. C) Cells were cultured for 24, 48 and $72 \mathrm{~h}$ and CCK-8 was performed to detect the cell viability. D) Cells were cultured for $48 \mathrm{~h}$ and EdU incorporation assay was performed. E) Flow cytometric detection of apoptosis in PC3 and DU145 cells with transfection of miR-877-5p mimic for $48 \mathrm{~h}$; experiments were independently repeated three times. ${ }^{* *} \mathrm{p}<0.01 v s$ NC mimic. PCa, prostate cancer; CCK-8, cell counting kit-8; EdU, 5-ethynyl-20-deoxyuridine. 


\section{Materials and Methods}

\section{Clinical tissues}

PCa tissues were obtained from 30 patients diagnosed in the department of TCM Surgery of Affiliated Hospital of Jiangxi University of Traditional Chinese Medicine (Nanchang, China). All the specimens were reviewed and verified by pathologists and immediately frozen in liquid nitrogen. All subjects gave their verbal informed consent for participation in the study. Ethical approval was obtained for all experimental procedures by the Ethical Committee of the Affiliated Hospital of Jiangxi University of Traditional Chinese Medicine

\section{Cell culture and transfection}

Human PCa cell lines (P4E6, LNCaP, PC3 and DU145) and prostate epithelial RWPE-1 cells were bought from Cell Resource Center of Shanghai Institute of Life Sciences (Shanghai, China) and cultured in DMEM medium with fetal bovine serum (Gibco, Rockville, MD, USA) at $37^{\circ} \mathrm{C}$ with $5 \% \mathrm{CO}_{2}$. Then, $\mathrm{PC} 3$ and DU145 cells were transfected with either miR-877-5p mimic, pcDNA3.1-sperm-specific antigen 2 (SSFA2) or their combinations using Lipofectamin 2000 (Invitrogen, Carlsbad, CA, USA).
The miR-877-5p mimic and pcDNA3.1-SSFA2 were synthesized by GenePharma (Shanghai, China).

\section{Luciferase reporter assay}

SSFA2 was predicted as a potential target of miR-877-5p through the TargetScan software (http://www.targetscan.org/). The binding site was identified by TargetScan and confirmed by a dual luciferase assay. The 3'UTR wild type (WT) or mutant type (MUT) sequence of SSFA2 gene was cloned into the vector pGL3 containing the luciferase reporter gene. Either the WT or MUT plasmids were transfected into PC3 and DU145 cells by Lipofectamin 2000 in combination with miR-877-5p mimic; $48 \mathrm{~h}$ post-transfection, the luciferase activity was measured using a dual-luciferase reporter assay system (Promega, Madison, WI, USA).

\section{RNA pull-down}

Cells were harvested and lysed in specific lysis buffer (Ambion, Austin, TX, USA). Following this, cell lysates were added with M-280 streptavidin magnetic beads (Sigma-Aldrich, St. Louis, MO, USA) coated with RNase-free BSA and yeast tRNA (Sigma-Aldrich) and incubated at $4{ }^{\circ} \mathrm{C}$ for $4 \mathrm{~h}$. The enrichment of SSFA2 in co-precipitated RNAs was determined using real-time quantitative PCR (RT-qPCR).
A

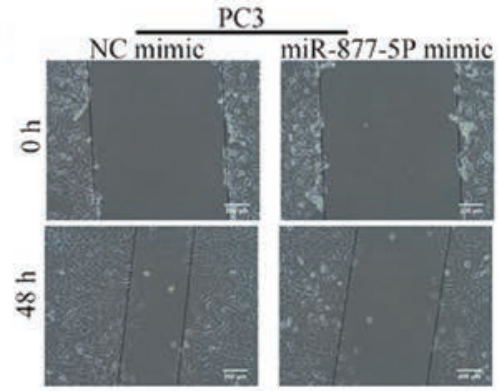

B

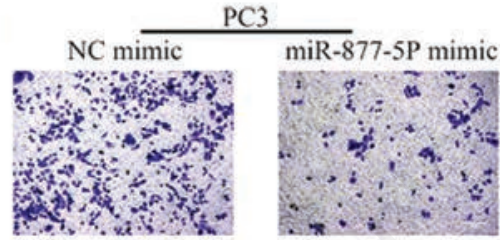

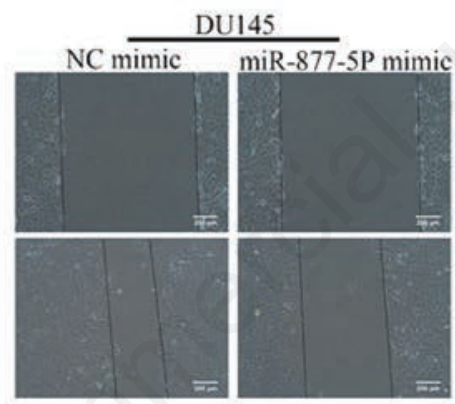
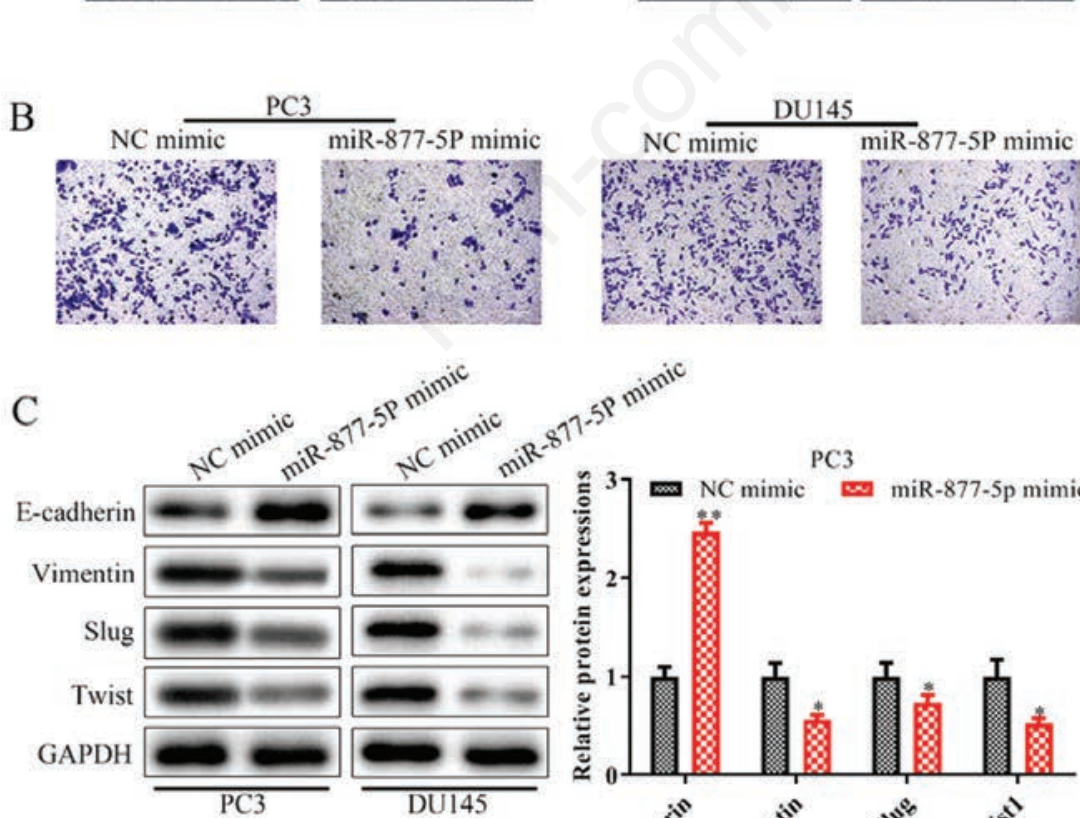<smiles>CC1CCCCC1</smiles>

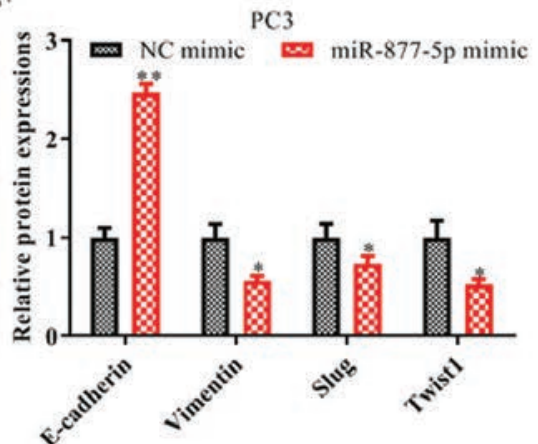

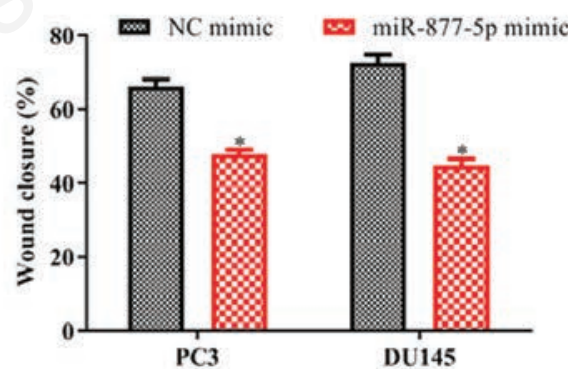
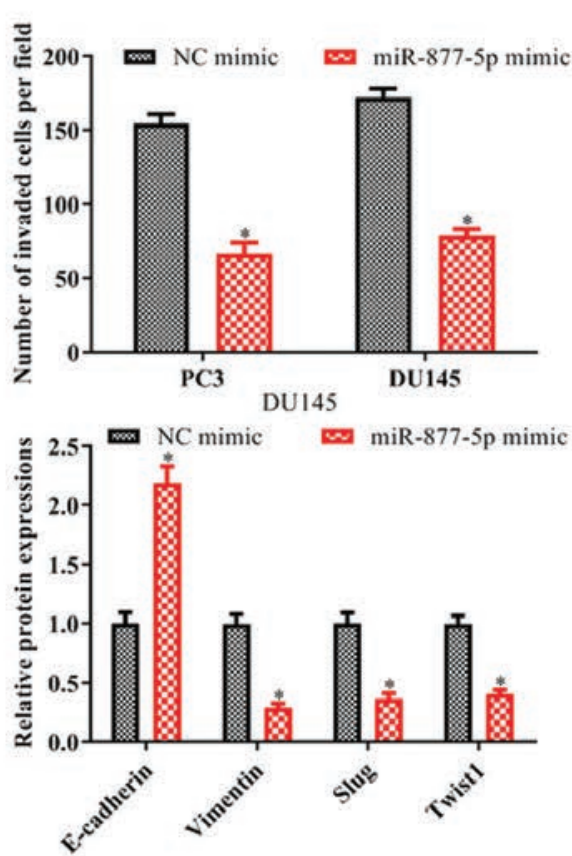

Figure 2. MiR-877-5p inhibits the metastasis of PCa cells. Cells were treated similarly as in Figure 1. Wound-healing assay (A), transwell invasion assay (B) and the expression of E-cadherin, Vimentin, Slug and Twist1 (C) in PC3 and DU145 cells with transfected with miR877-5p mimic for $48 \mathrm{~h}$. Experiments were independently repeated three times. NC, negative control. ${ }^{*} \mathrm{p}<0.05$ vs NC mimic. 


\section{RT-qPCR}

Total RNA was isolated from cells with TRIzol reagent (Invitrogen). The SuperScript RT kit (Fermentas, Ottawa, Canada) was adopted to synthesize cDNA. The RT-qPCR assay was then performed on the Applied Biosystems 7500 Real-Time PCR system (Applied Biosystems, Foster City, USA) by utilizing SYBR Green PCR Master Mix (Takara Bio, Otsu, Japan). U6 and GAPDH were used as internal references, respectively. RNA expression was assessed using $2^{-\Delta \Delta C t}$ method. The primers were shown in Table 1.

\section{Western blot}

Total protein were obtained from cells lysed with RIPA buffer (Invitrogen, USA), and protein concentration was measured using a bicinchoninic acid (BCA) assay, separated by $10 \%$ sodium dodecyl sulfate-polyacrylamide gel electrophoresis (SDS-PAGE), and electro-transferred to polyvinylidene fluoride (PVDF) membranes. The membranes were blocked with 5\% non-fat milk for $1 \mathrm{~h}$. Membranes were immunoblotted with indicated primary antibodies at $4^{\circ} \mathrm{C}$ overnight. After washing with TBS with Tween-20, cells were probed with HRP-conjugated secondary antibody for another $2 \mathrm{~h}$. The protein bands were visualized by ECL kit (Millipore,
Bedford, MA, USA) and quantified using ImageJ software. GAPDH was used as an internal reference. The specific primary antibodies were as follows: anti-E-cadherin (cat: ab231303, 1:1000; Abcam, Cambridge, UK), anti-Vimentin (ab20346, 1:1000, Abcam), anti-slug (ab27568, 1:1000, Abcam), anti-twist (ab175430, 1:1000, Abcam), anti-SSFA2 (ab195334, 1:1000, Abcam) and anti-GAPDH (ab9485, 1:1000, Abcam). The secondary antibodies were as follows: goat anti-rabbit IgG H\&L (HRP) (ab97080, 1:20000, Abcam) and goat anti-mouse IgG H\&L (HRP) (ab97040, 1:20000, Abcam)

Table 1. The primer sequences used in qRT-PCR.

\begin{tabular}{lc}
\hline miR-877-5p forward & 5'-GTAGAGGAGATGGCGCAGGG-3' \\
miR-877-5p reverse & 5'-GTAGAGGAGATGGCGCAGGG-3' \\
\hline SSFA2 forward & 5'-CGGAGACGGAGGATCTGTC-3' \\
SSFA2 reverse & 5'-AGTGAGGCTCCCAAAGGTGT-3' \\
\hline U6 forward & 5-CTTCGGCAGCACATATACT-3 \\
U6 reverse & 5-AAAATATGGAACGCTTCACG-3 \\
\hline GAPDH forward & 5-CGGAGTCAACGGATTTGGTCGTAT-3 \\
GAPDH reverse & 5 -AGCCTTCTCCATGGTGGTGAAGAC-3
\end{tabular}

A
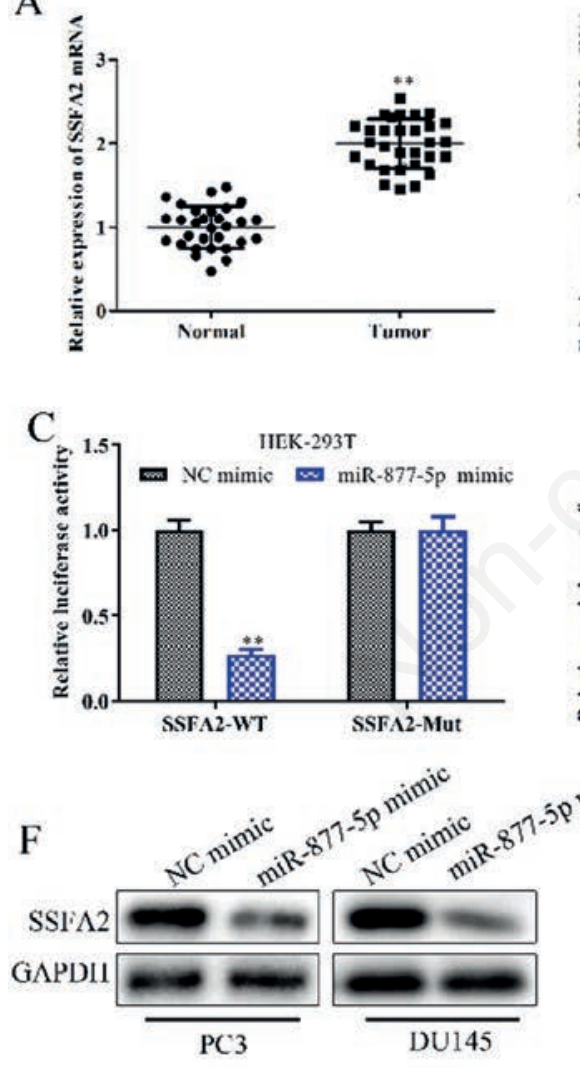
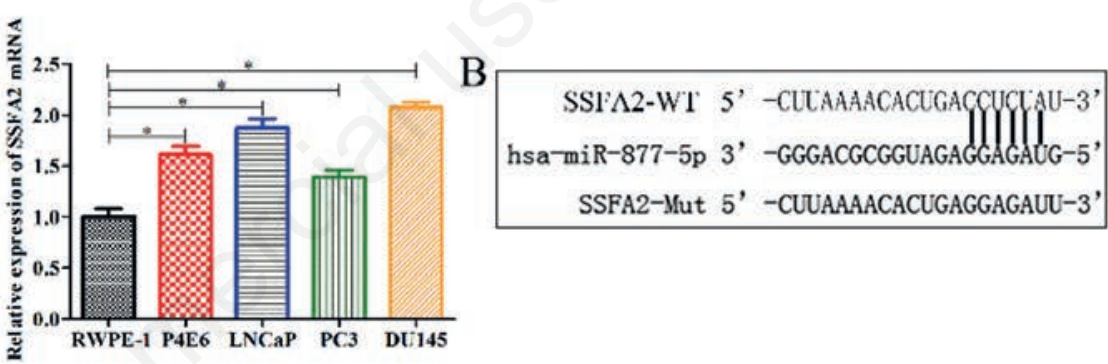

Bio-NC

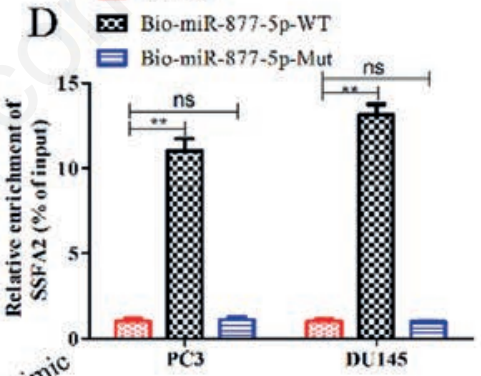

E
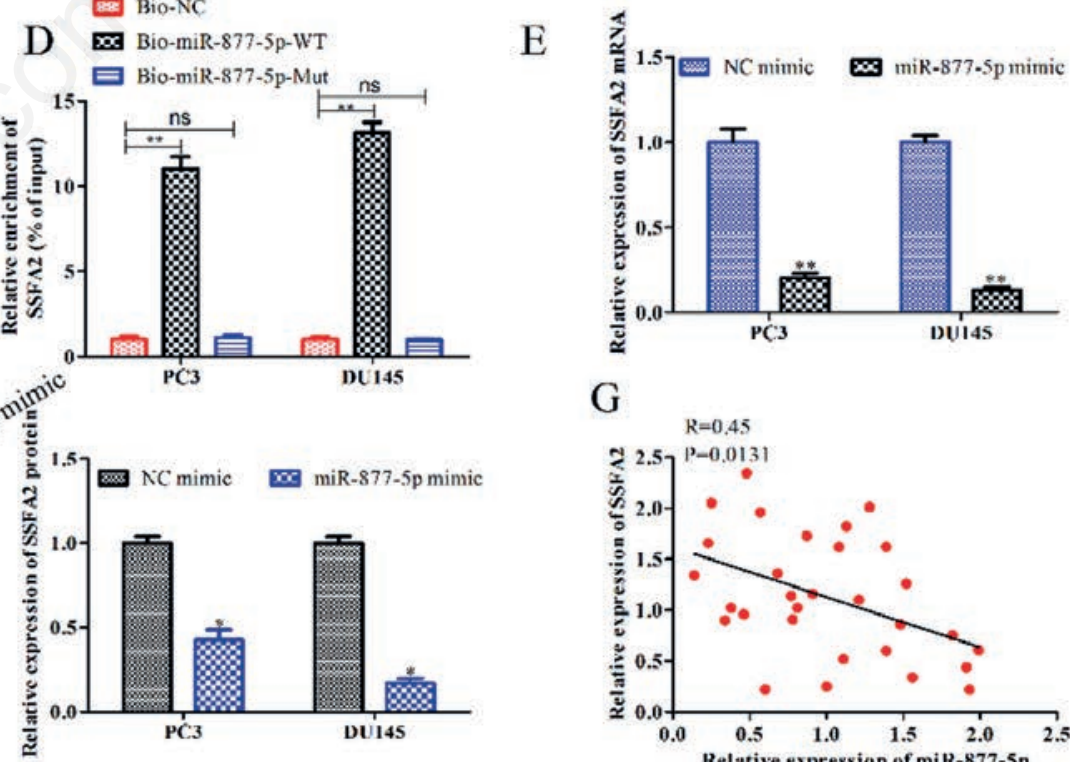

G

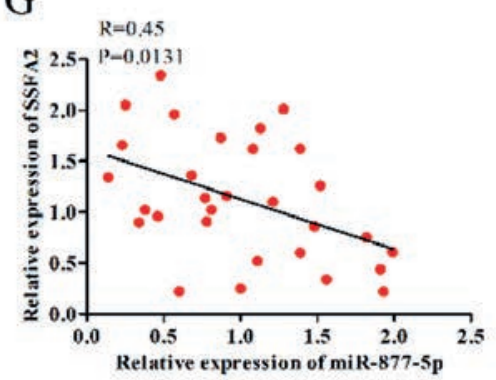

Figure 3. SSFA2 is a target of miR-877-5p. A) SSFA2 mRNA expression in PCa cell lines; ${ }^{*} \mathrm{p}<0.05$ vs RWPE-1 cells. B) Predicted binding sites of miR-877-5p in SSFA2 3'-UTR. C) Luciferase assay results from miR-877-5p-overexpressed cells transfected with SSFA2-3'-UTR reporter plasmids; ${ }^{*} \mathrm{p}<0.05 v s$ NC mimic. D) Verification of the relationship between miR-877-5p and SSFA2 by RNA pull-down assay; ${ }^{*} \mathrm{p}<0.05$ vs Bio-NC; \#p<0.05 vs Bio-miR-877-5p WT. E) SSFA2 mRNA and protein levels in PC3 and DU145 cells transfected with miR-877-5p mimic; ${ }^{*}$ p $<0.05$ vs NC mimic. Experiments were independently repeated three times. WT, wild type. 


\section{Proliferation assay}

Cell proliferation was assessed by CCK-8 and EdU incorporation assays (Dojindo, Kumamoto, Japan). For CCK-8, cells $\left(3 \times 10^{5}\right)$ were inoculated into each well of 96-well plates and cultured for $0,24,48$ and $72 \mathrm{~h}$. Then, $10 \mu \mathrm{L}$ of CCK-8 reagent were added and cells were incubated for additional $4 \mathrm{~h}$ at $37^{\circ} \mathrm{C}$. At last, optical density was determined at a wavelength of $450 \mathrm{~nm}$ by a microplate reader. For EdU assay, EdU incorporation assay using KFluor488 EdU kit (Invitrogen) according to the manufacturer's instructions.

\section{Apoptosis detection}

PC3 and DU145 cells were cultured in a 6-well plate and labeled with Annexin V/PI fluorescent double staining (BD Biosciences, Bedford, MA, USA) for $30 \mathrm{~min}$ as previously described. ${ }^{22}$ The apoptotic cells were assessed using flow cytometry (FACScan; BD Biosciences) using FlowJo 10.06 software (FlowJo LLC, Ashland, OR, USA).

\section{Migration assay}

Wound-healing assay was used to evaluate cell migration activity as previously described. ${ }^{23}$ Briefly, transfected cells were wounded using a sterile micropipette tip, and photographed under a microscope (Olympus, Tokyo, Japan) at $0 \mathrm{~h}$ and $48 \mathrm{~h}$ after scratching.

\section{Invasion assay}

Cell invasion assay was performed using a transwell chamber (BD Biosciences) as previously described. ${ }^{24}$ Cells were seeded in the upper wells, while the lower wells were filled with culture media with $10 \%$ FBS. After 12-h incubation, the cells which did not invaded to the lower chamber were removed. Cells were fixed with $4 \%$ paraformaldehyde and stained with $1 \%$ crystal violet. Representative images were captured, and the numbers of cells were counted in five random fields under a light microscope at $\times 200$ magnification and analyzed using Image J software.

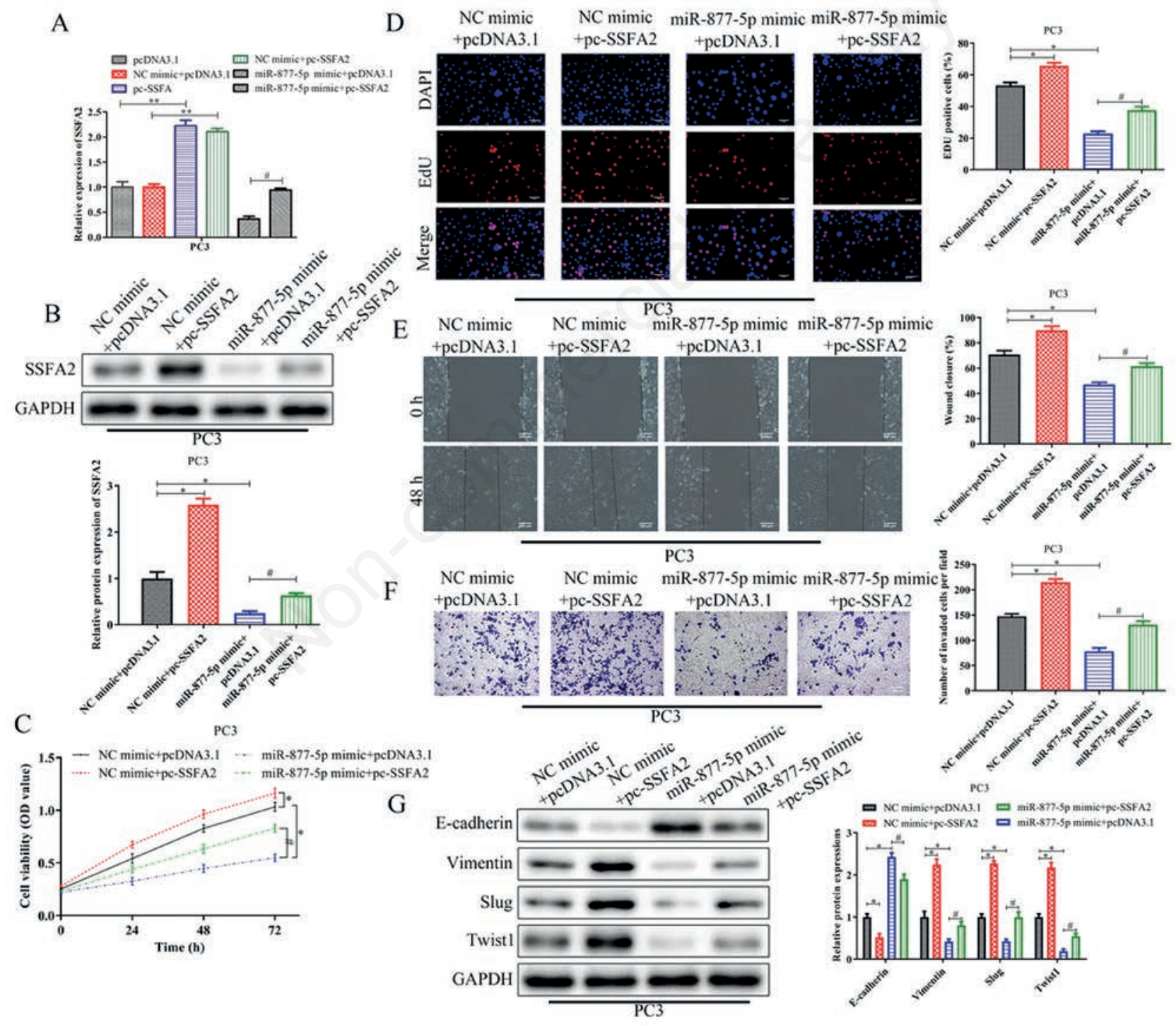

Figure 4. MiR-877-5p exhibits tumor-suppressive roles via targeting SSFA2. PC3 cells were transfected with miR-877-5p mimic and/or pc-SSFA2. A,B) SSFA2 mRNA and protein levels. C) CCK-8 cell viability. D) EdU incorporation assay. E) wound-healing assay. F) Transwell invasion assay. G) E-cadherin, Vimentin, Slug and Twist1 expression; experiments were independently repeated three times. NC, negative control; ${ }^{*} \mathrm{p}<0.05$ vs NC mimic+pcDNA3.1; \# $\mathrm{p}<0.05$ vs miR-877-5p mimic+pcDNA3.1. 


\section{Statistical analysis}

Experiments were independently repeated three times. All data were statistically analyzed by SPSS 19.0 software (IBM Corp.) and expressed as mean \pm SD. Student's $t$-test and one-way analysis of variance (ANOVA) were used to assess the difference between groups; $\mathrm{p}<0.05$ was considered statistically significant.

\section{Results}

\section{miR-877-5p suppresses PCa cell proliferation and induces apoptosis}

As shown in Figure 1A, the expression levels of miR-877-5p were significantly decreased in PCa tissues and cell lines (P4E6, LNCaP, PC3 and DU145) $(\mathrm{p}<0.01)$ compared with those in the normal tissues and RWPE-1 cells. Subsequently, to examine the effect of miR-877-5p on the PCa cell proliferation, we overexpressed the miR-877-5p in the PC3 and DU145 cells, since the miR-877-5p was lower expressed in these cells, by using miR-877$5 p$ mimic. The miR-877-5p mimic successfully increased the expression of miR-877-5p in these cells (Figure 1B). More importantly, CCK-8 and EdU assays demonstrated that miR-877-5p mimic led to a markedly decrease $(\mathrm{p}<0.01)$ in PCa cell proliferation (Figure 1C-1D). In contrast, the apoptotic rate was enhanced in miR-877-5p mimic-treated PCa cells (Figure 1E).

\section{miR-877-5p inhibits the metastasis of PCa cells}

Cell migration and invasion were evaluated by wound-healing assay and transwell invasion assay in PCa cells. As shown in Figure 2A, the wound-healing assay indicated that ectopic expression of miR-877-5p markedly attenuated the cell migratory capacityof PCa cells $(p<0.05)$. The transwell chamber assay showed that invaded cells transfected with miR-877-5p mimic significantly less than those in NC mimic group (Figure 2B). On the other hand, the epithelial-mesenchymal transition (EMT) process is known to facilitate the tumorigenesis ${ }^{25}$ by affecting cancer cell invasion and metastasis..$^{26,27}$ It should be noted that EMT is characterized by loss of the epithelial characteristics, including reduced E-cadherin expression and increased mesenchymal markers, such as vimentin and $\mathrm{N}$-cadherin. ${ }^{28,29}$ Furthermore, the EMT process is regulated by a set of transcription factors, including Slug and Twist. ${ }^{30}$ As presented in Figure 2C, overexpression of miR-877-5p notably increased the protein levels of E-cadherin, whereas decreased the expression of Vimentin, Slug and Twistl in the PCa cells.

\section{SSFA2 is a target of miR-877-5p}

Meanwhile, mRNA expression of SSFA2 was markedly upregulated in PCa tissues and cells, when compared with normal tissues and RWPE-1 cells, respectively (Figure 3A). The target sites of SSFA 2 and miR-877-5p was determined by TargetScan. The putative binding sequences of miR-877-5p on the 3'-UTR promoter of SSFA2 were presented in Figure 3B. The luciferase reporter assay was carried out and the results showed that the overexpression of miR-877-5p significantly decreased the luciferase activity of wildtype SSFA2-3'-UTR in PC3 and DU145 cells ( $\mathrm{p}<0.01$; Figure 3C). RNA pull-down assay suggested the enrichment of SSFA2 by biotinylated miR-877-5p, further verifying their interaction $(p<0.01$; Figure 3D). In addition, overexpression of miR-877-5p suppressed the mRNA and protein expression of SSFA2 in $\mathrm{PCa}$ cells $(\mathrm{p}<0.01$; Figure $3 \mathrm{E})$.

\section{miR-877-5p exhibits tumor-suppressive roles via target- ing SSFA2}

To further identify the role of SSFA2 in mediating the effect of
miR-877-3p, we overexpressed the SSFA2 by using pc-SSFA2 in PCa cells transfected with miR-877-3p mimic. The upregulation efficiency of SSFA2 in PCa cells was confirmed by RT-qPCR (Figure 4A) and Western blot (Figure 4B). Pathologically, enforced expression of SSFA2 promoted the cell proliferation (Figure 4 C,D), migration (Figure 4E) and invasion (Figure 4F), while led to a remarkable downregulation of E-cadherin expression, and an upregulation of Vimentin, Slug and Twist1 expression (Figure 4G), in the miR-877-5p mimic-treated cells. These results indicated that SSFA2 functioned as a mediator in linking the beneficial effects of miR-877-5p overexpression to the PCa progression.

\section{Discussion}

In recent years, dysregulation of miRNA-controlled physiological homeostasis has been implicated in the carcinogenesis of PCa. ${ }^{31}$ In the present study, we determined expression levels of miR-877-5p in PCa cells, while investigated its functional roles and underlying molecular mechanisms involved in the pathogenesis of PCa. Our research might provide novel insights into the pathogenesis and therapeutic strategies for PCa.

Studies have increasingly suggested that miRNAs govern their biological functions in the development of tumors, including PCa. For example, $\mathrm{Wu}$ et al. indicated that miR-302a increases the chemo-resistance to paclitaxel in PCa cells. ${ }^{32}$ miR-153 expression is also an independent prognostic factor in patients with $\mathrm{PCa}$, since its expression is associated with aggressive pathological parameters in PCa tissues. ${ }^{32}$ Yang et al. illustrated that knockdown of miR139-5p expression promotes EMT process in PCa progression. ${ }^{33} \mathrm{Ji}$ et al. manifested that miR-589-5p functions as a tumor inhibitor by affecting tumor migration, invasion and apoptosis. ${ }^{34}$ miR-877-5p has been widely studied in various cancers. For instance, MiR$877-5 \mathrm{p}$ suppresses cell growth, migration and invasion by targeting cyclin dependent kinase 14 in hepatocellular carcinoma. ${ }^{19}$ In glioblastoma, miR-877-5p is regulated by lncRNA TRG-AS1 and promotes tumor cell proliferation via targeting suppressor of Zeste 12 (SUZ12). ${ }^{20}$ Herein, we found that miR-877-5p was dramatically downregulated in PCa cell lines. The overexpression of miR-877$5 \mathrm{p}$ can inhibit the proliferation, migration and invasion of $\mathrm{PCa}$ cells, and the EMT process could also be suppressed. Hence, miR877-5p can be considered as a potential tumor suppressor in the progression of $\mathrm{PCa}$, which may inhibit tumor metastasis by suppressing the EMT process of PCa.

MicroRNAs are involved in the regulation of eukaryotic gene expression by post-transcriptional regulation through binding to 3'-UTR of mRNA of their target genes. ${ }^{35}$ By bioinformatics analysis, we demonstrated that SSFA2 had the potential target sites of miR-877-5p. SSFA2, also known as ITPR interacting domain containing 2 (ITPRID2), is originally identified in human colon cancer cells. ${ }^{36}$ Several lines of evidence suggest that SSFA2 serves as a well-known oncogene. For example, Okayama et al. reported that high phosphorylation levels of SSFA2 predicted poor survival in lung adenocarcinoma ${ }^{37} \mathrm{~A}$ recent study indicated that the inhibition of SSFA2 reduced the cell proliferation, regulated $\mathrm{G}_{1} / \mathrm{S}$ transition and induced apoptosis in glioma. ${ }^{38}$ Thus, exploration of the potential of SSFA2 as a biomarker or therapeutic target gene would provide a novel insight into PCa treatment. The current study found that the expression of miR-877-5p and SSFA2 was negatively correlated in PCa cells. Rescue experiments verified that the overexpression of SSFA2 was sufficient for reversing the anti-tumor effects of miR-877-5p on PCa progression. Similar findings were found in the study of Zhu et al., who found that miR-363-3p was down-regulated in oral squamous cell carcinoma (OSCC), and miR-363-3p inhibited the proliferation and invasion of OSCC cells 
by inhibiting the expression of its target SSFA2. ${ }^{39}$ The discovery of miR-877-5p/SSFA2 axis in this research may provide a new diagnostic and therapeutic strategy for PCa. However, several limitations were also existed in our study. Firstly, the in vivo experiments are lacked. Secondly, the underlying mechanisms for SSFA2 in PCa development are not intensively studied. Lastly, whether other miR-877-5p's targets are involved in the miR-877-5p-controlled $\mathrm{PCa}$ is still elusive.

In summary, our study for the first time implied that miR-877$5 p$ suppressed SSFA2 expression, thereby inhibiting the proliferation and metastasis, while inducing the apoptosis of PCa cells, suggesting that miR-877-5p and SSFA2 may be novel therapeutic biomarkers for $\mathrm{PCa}$.

\section{References}

1. Welch HG, Albertsen PC. Reconsidering prostate cancer mortality - The future of PSA screening. N Engl J Med 2020;382:1557-63.

2. Boulos S, Mazhar D. The evolving role of chemotherapy in prostate cancer. Future Oncol 2017;13:1091-5.

3. Sebesta EM, Anderson CB. The surgical management of prostate cancer. Semin Oncol 2017;44:347-57.

4. Shevach J, Chaudhuri P, Morgans AK. Adjuvant therapy in high-risk prostate cancer. Clin Adv Hematol Oncol 2019; 17:45-53.

5. Chistiakov DA, Myasoedova VA, Grechko AV, Melnichenko AA, Orekhov AN. New biomarkers for diagnosis and prognosis of localized prostate cancer. Semin Cancer Biol 2018;52:9-16.

6. Rupaimoole R, Slack FJ. MicroRNA therapeutics: towards a new era for the management of cancer and other diseases. Nat Rev Drug Discov 2017;16:203-22.

7. Lun W, Wu X, Deng Q, Zhi F. MiR-218 regulates epithelialmesenchymal transition and angiogenesis in colorectal cancer via targeting CTGF. Cancer Cell Int 2018;18:83.

8. Wang Y, Tian Y. miR-206 Inhibits cell proliferation, migration, and invasion by targeting BAG3 in human cervical cancer. Oncol Res 2018;26:923-31.

9. Wang Z, Zhao Z, Yang Y, Luo M, Zhang M, Wang X, et al. MiR-99b-5p and miR-203a-3p function as tumor suppressors by targeting IGF-1R in gastric cancer. Sci Rep 2018;8:10119.

10. Bjartell A. New hope in prostate cancer precision medicine? miRNA replacement and epigenetics. Clin Cancer Res 2019;25:2679-81.

11. Huh JH, Kim TH, Kim K, Song JA, Jung YJ, Jeong JY, et al. Dysregulation of miR-106a and miR-591 confers paclitaxel resistance to ovarian cancer. Br J Cancer 2013;109:452-61.

12. Liu C, Kelnar K, Liu B, Chen X, Calhoun-Davis T, Li H, et al. The microRNA miR-34a inhibits prostate cancer stem cells and metastasis by directly repressing CD44. Nat Med 2011;17:211-5.

13. Xing R. miR-3648 Promotes prostate cancer cell proliferation by inhibiting adenomatous polyposis coli 2. J Nanosci Nanotechnol 2019;19:7526-31.

14. Zhang X, Zhou J, Xue D, Li Z, Liu Y, Dong L. MiR-515-5p acts as a tumor suppressor via targeting TRIP13 in prostate cancer. Int J Biol Macromol 2019;129:227-32.

15. Wang F, Chang JT, Kao CJ, Huang RS. High expression of miR-532-5p, a tumor suppressor, leads to better prognosis in ovarian cancer both in vivo and in vitro. Mol Cancer Ther 2016;15:1123-31

16. Zhang L, Li C, Cao L, Li H, Zou H, Li H, et al. microRNA877 inhibits malignant progression of colorectal cancer by directly targeting MTDH and regulating the PTEN/Akt path- way. Cancer Manag Re. 2019;11:2769-81.

17. Pafundi PC, Caturano A, Franci G. Comment on: MiR-877-5p suppresses cell growth, migration and invasion by targeting cyclin dependent kinase 14 and predicts prognosis in hepatocellular carcinoma. Eur Rev Med Pharmacol Sci 2018;22:4401-2.

18. Xiong DD, Dang YW, Lin P, Wen DY, He RQ, Luo DZ, et al. A circRNA-miRNA-mRNA network identification for exploring underlying pathogenesis and therapy strategy of hepatocellular carcinoma. J Transl Med 2018;16:220.

19. Yan TH, Qiu C, Sun J, Li WH. MiR-877-5p suppresses cell growth, migration and invasion by targeting cyclin dependent kinase 14 and predicts prognosis in hepatocellular carcinoma. Eur Rev Med Pharmacol Sci 2018;22:3038-46.

20. Xie H, Shi S, Chen Q, Chen Z. LncRNA TRG-AS1 promotes glioblastoma cell proliferation by competitively binding with miR-877-5p to regulate SUZ12 expression. Pathol Res Pract 2019;215:152476.

21. Liang J, Zhang S, Wang W, Xu Y, Kawuli A, Lu J, et al. Long non-coding RNA DSCAM-AS1 contributes to the tumorigenesis of cervical cancer by targeting miR-877-5p/ATXN7L3 axis. Biosci Rep 2020;40:BSR20192061.

22. Yun JM, Kweon MH, Kwon H, Hwang JK, Mukhtar H. Induction of apoptosis and cell cycle arrest by a chalcone panduratin A isolated from Kaempferia pandurata in androgenindependent human prostate cancer cells PC3 and DU145. Carcinogenesis 2006;27:1454-64.

23. Wang X, Decker CC, Zechner L, Krstin S, Wink M. In vitro wound healing of tumor cells: inhibition of cell migration by selected cytotoxic alkaloids. BMC Pharmacol Toxicol 2019;20:4.

24. You F, Luan H, Sun D, Cui T, Ding P, Tang H, et al. miRNA106a promotes breast cancer cell proliferation, clonogenicity, migration, and invasion through inhibiting apoptosis and chemosensitivity. DNA Cell Biol 2019;38:198-207.

25. Tam WL, Weinberg RA. The epigenetics of epithelial-mesenchymal plasticity in cancer. Nat Med 2013;19:1438-49.

26. Pan JJ, Yang MH. The role of epithelial-mesenchymal transition in pancreatic cancer. J Gastrointest Oncol 2011;2:151-6.

27. Pastushenko I, Blanpain C. EMT Transition states during tumor progression and metastasis. Trends Cell Biol 2019;29:212-26.

28. Gonzalez DM, Medici D. Signaling mechanisms of the epithelial-mesenchymal transition. Sci Signal 2014;7:re8.

29. Ye X, Weinberg RA. Epithelial-mesenchymal plasticity: A central regulator of cancer progression. Trends Cell Biol 2015;25:675-86.

30. Nieto MA. Epithelial plasticity: a common theme in embryonic and cancer cells. Science 2013;342:1234850.

31. Massillo C, Dalton GN, Farre PL, De Luca P, De Siervi A. Implications of microRNA dysregulation in the development of prostate cancer. Reproduction 2017;154:R81-97.

32. Wu Y, Hu L, Qin Z, Wang X. MicroRNA302a upregulation mediates chemoresistance in prostate cancer cells. Mol Med Rep 2019;19:4433-40.

33. Yang B, Zhang W, Sun D, Wei X, Ding Y, Ma Y, et al. Downregulation of miR-139-5p promotes prostate cancer progression through regulation of SOX5. Biomed Pharmacother 2019;109:2128-35.

34. Ji L, Jiang X, Mao F, Tang Z, Zhong B. miR5895p is downregulated in prostate cancer and regulates tumor cell viability and metastasis by targeting CCL5. Mol Med Rep 2019;20:1373-82.

35. Wang Z, Liu Y. Predicting functional MicroRNA-mRNA interactions. Methods Mol Biol 2017;1580:117-26.

36. Fujimoto T, Koyanagi M, Baba I, Nakabayashi K, Kato N, 
Sasazuki T, et al. Analysis of KRAP expression and localization, and genes regulated by KRAP in a human colon cancer cell line. J Hum Genet 2007;52:978-84.

37. Okayama A, Kimura Y, Miyagi Y, Oshima T, Oshita F, Ito H, et al. Relationship between phosphorylation of sperm-specific antigen and prognosis of lung adenocarcinoma. J Proteomic. 2016;139:60-6.

38. Zhu A, Li X, Wu H, Miao Z, Yuan F, Zhang F, et al. Molecular mechanism of SSFA2 deletion inhibiting cell proliferation and promoting cell apoptosis in glioma. Pathol Res Pract 2019;215:600-6.

39. Zhu L, Zhang L, Tang Y, Zhang F, Wan C, Xu L, et al. MicroRNA-363-3p inhibits tumor cell proliferation and invasion in oral squamous cell carcinoma cell lines by targeting SSFA2. Exp Ther Med 2021;21:549.

Received for publication: 11 March 2021. Accepted for publication: 19 July 2021.

This work is licensed under a Creative Commons Attribution-NonCommercial 4.0 International License (CC BY-NC 4.0)

(C) Copyright: the Author(s), 2021

Licensee PAGEPress, Italy

European Journal of Histochemistry 2021; 65:3243

doi:10.4081/ejh.2021.3243 